\title{
Radio Frequency Transistors Using Aligned Semiconducting Carbon Nanotubes with Current-Gain Cutoff Frequency and Maximum Oscillation Frequency Simultaneously Greater than \\ $70 \mathrm{GHz}$
}

Yu Cao, ${ }^{1, \S}$ Gerald J. Brady, ${ }^{2,}{ }^{\S}$ Hui Gui, ${ }^{3}$ Chris Rutherglen, ${ }^{4}$ Michael S. Arnold, ${ }^{2}$ and Chongwu Zhou ${ }^{1,3, *}$

${ }^{1}$ Department of Electrical Engineering, University of Southern California, Los Angeles, California 90089, United States

${ }^{2}$ Department of Materials Science and Engineering, University of Wisconsin-Madison, Madison, Wisconsin 53706, United States

${ }^{3}$ Department of Chemical Engineering and Materials Science, University of Southern California, Los Angeles, California 90089, United States

${ }^{4}$ Carbonics Inc., Marina del Rey, California 90292, United States

${ }^{\S}$ These authors contributed equally to this work.

*To whom correspondence should be addressed: chongwuz@usc.edu. 


\section{S1: Transfer curves of self-aligned T-shape gate nanotube radio-frequency (RF) transistors under various drain-to-source biases}

Figure S1(a) shows the transfer curves of a representative self-aligned T-shape gate nanotube RF transistor under various drain-to-source biases $\left(V_{D S}\right)$ from -0.1 to $-1.6 \mathrm{~V}$ with a step of $-0.3 \mathrm{~V}$. Figure $\mathrm{S} 1(\mathrm{~b})$ shows the corresponding on/off ratio at various $V_{D S}$. The on/off ratio decreases from $\sim 2000$ to $\sim 50$ with the increase of $V_{D S}$ from -0.1 to $-1.6 \mathrm{~V}$, suggesting that the low on/off ratio for the T-gate RF transistors measured at $V_{D S}=-1.5 \mathrm{~V}$ might be caused by the drain-induced barrier lowering (DIBL) effect.

(a)

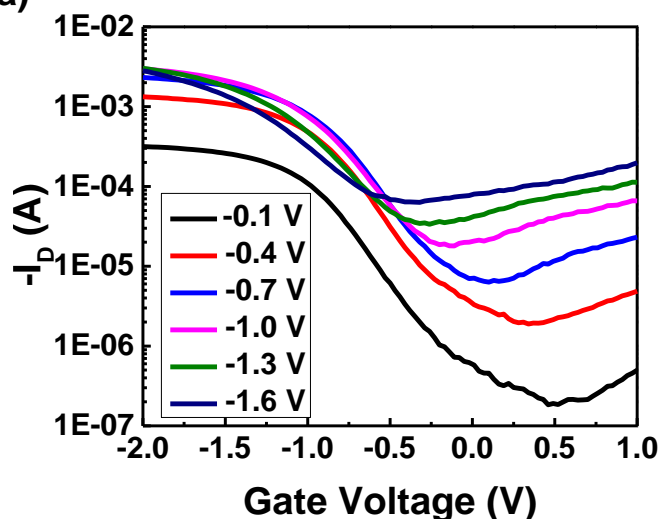

(b)

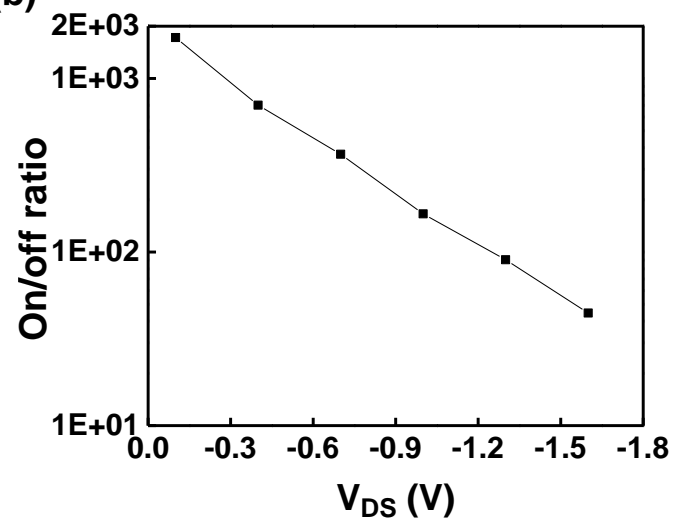

Figure S1 (a) Transfer curves of a representative self-aligned T-shape gate nanotube RF transistor under various drain-to-source biases $\left(V_{D S}\right)$ from -0.1 to $-1.6 \mathrm{~V}$ with a step of 0.3 V. (b) Corresponding on/off ratio at various $V_{D S}$ extracted from (a). 


\section{S2: Device de-embedding structure}

Figure S2 shows the open and short structures for the device de-embedding structure (DDS). The DDS would remove only the parasitics from the bonding pads but without removing the capacitances associated with the gate, and reveal the performance for real applications.

(a)

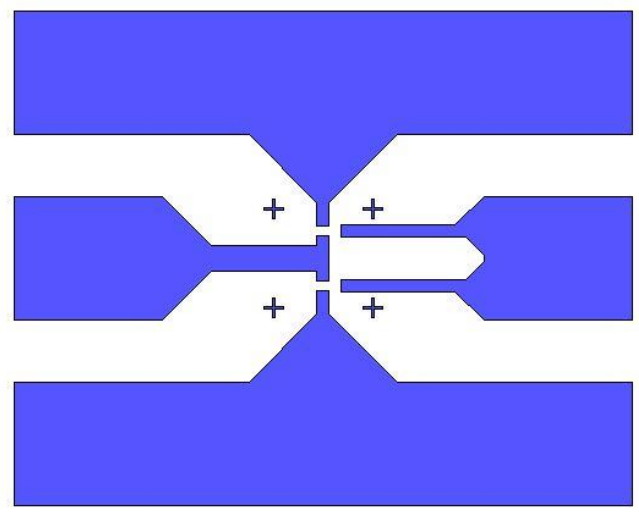

(b)

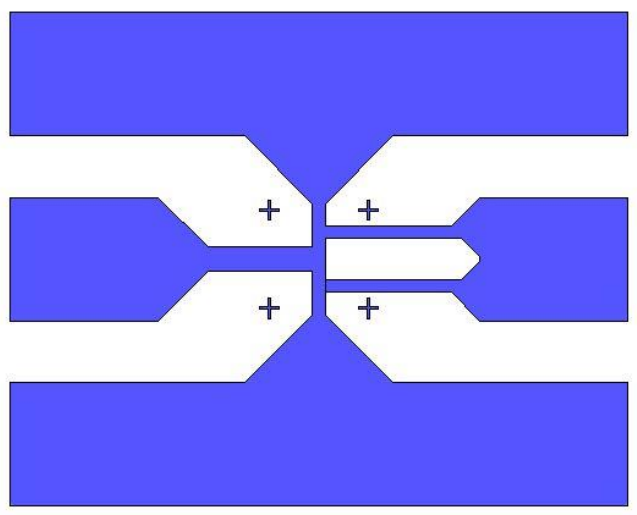

Figure S2 (a) Open structure for DDS. (b) Short structure for DDS. 


\section{S3: Intrinsic de-embedding structure}

Figure S3 shows the open and short structures for the intrinsic de-embedding structure (IDS). IDS would remove the parasitics from the bonding pads and the fringe capacitances associated with the gate, and reveal the performance upper-limit of material properties.

(a)

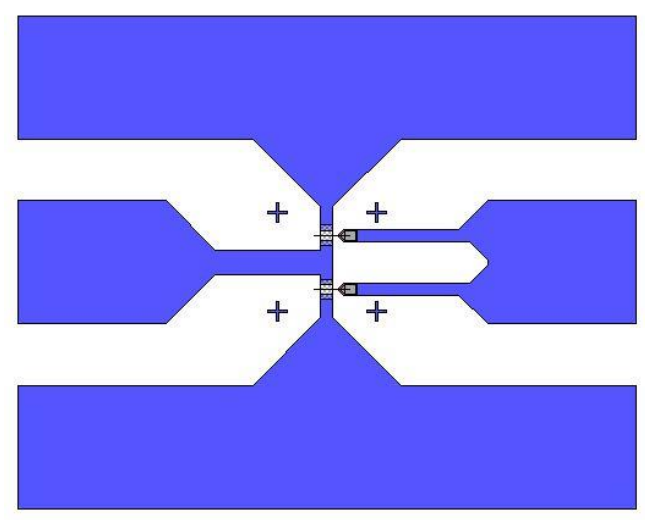

(b)

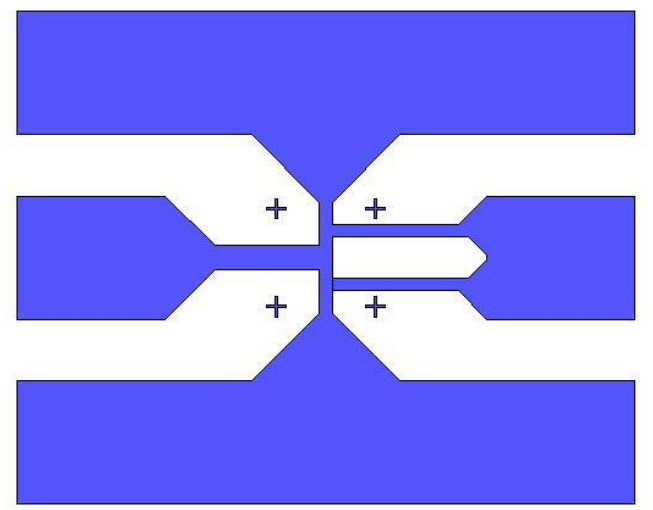

Figure S3 (a) Open structure for IDS. (b) Short structure for IDS. 


\section{S4: De-embedding process for the linearity measurement}

In order to de-embed out the losses from the wires and connectors for the linearity measurement, we carried out the single-tone test for an on-chip short structure (Figure S2) at frequencies of 1, 4, 8, 12 and $16 \mathrm{GHz}$. The results for the single-tone test are shown in Figure S4(a). The losses for the wires and connectors are extracted by subtracting input power from the output power, and the results are plotted in Figure S4(b).

(a)

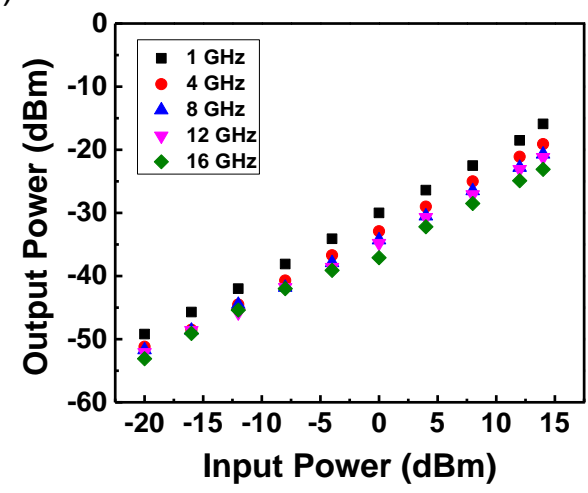

(b)

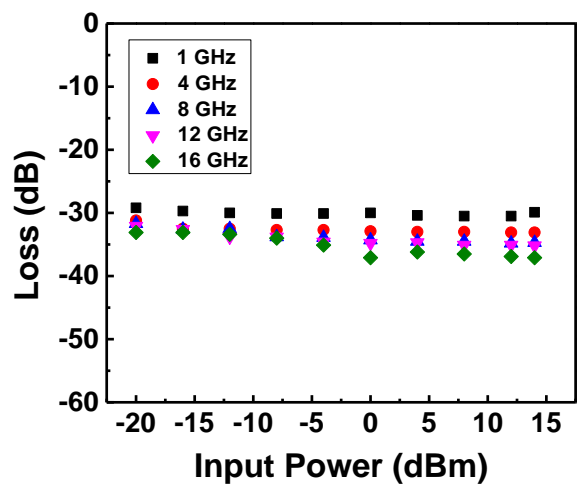

Figure S4 (a) Output power vs. input power curves for the single-tone test of an on-chip short structure at frequencies of $1,4,8,12$ and $16 \mathrm{GHz}$. (b) Losses from wires and connectors at frequencies of $1,4,8,12$ and $16 \mathrm{GHz}$. 